\title{
Cross Sectional Study Comparing Peripheral Blood Smear and Rapid Antigen Detection Test in Diagnosis of Malaria Conducted During Monsoon and Post Monsoon Period at Ballari, India
}

\author{
B. Vishalakshi", J. Mariraj, S. Liba and S. Krishna \\ ${ }^{1}$ Department of Microbiology, Vijayanagar Institute of Medical Sciences, Ballari, \\ Karnataka, India \\ *Corresponding author
}

A B S T R A C T

\begin{tabular}{|l|}
\hline Ke y w o r d s \\
$\begin{array}{l}\text { Peripheral blood } \\
\text { smear, Rapid } \\
\text { antigen, Detection } \\
\text { test }\end{array}$ \\
\hline Article Info \\
\hline $\begin{array}{l}\text { Accepted: } \\
\text { 04 January } 2018 \\
\text { Available Online: } \\
\text { 10 February } 2018\end{array}$ \\
\hline
\end{tabular}

Malaria is major public health issue and Ballari is an endemic area. Peripheral blood smear examination (PBS) is done routinely. However, PBS during febrile period is essential for demonstration of parasite. In most cases, sample collection during afebrile period or at inappropriate timings results in smear negative reports. Hence, rapid antigen detection tests would be ideal or can be a supportive test to early diagnosis of malaria. To compare PBS examination with rapid antigen detection tests (RDT) and estimate prevalence of malaria. Patients with PUO attending VIMS, June-October 2016 were included in cross sectional study. $1 \mathrm{ml}$ venous blood collected into EDTA bulbs and subjected to antigen detection using commercially available kits (SD Bioline) and then PBS was prepared and stained using Leishman stain. Among the 2979 samples, 1616 (54.24\%) and 1363 (45.75\%) were tested during Monsoon and post-monsoon period respectively. Among the 1616 samples, $12(0.74 \%)$ were positive, of which $12(100 \%)$ were tested positive by RDT, while 3(25\%) samples were positive by PBS, while all 1363 samples were negative. The age group affected was 20-40 years (75\%) with sex ratio of 3:1 (M: F) showing male preponderances (75\%). Geographic prevalence showed high positive trend in urban Ballari (50\%). The sensitivity $(75 \%)$ and specificity $(100 \%)$ was with RDT compared with PBS. Study revealed declining trend of malaria in 2016. Rapid antigen detection kits are more specific and sensitive in detection of the malarial parasites especially in endemic areas even with low parasitaemia.

\section{Introduction}

Malaria is the parasitic disease caused by an obligate intracellular protozoan belonging to genus Plasmodium. Malaria is the $5^{\text {th }}$ most common cause of death due to infectious disease worldwide. World Malaria Report
2015, estimated 214 million cases of malaria globally and 4.38 lakh malaria deaths, representing a decrease in malaria cases and deaths of $37 \%$ and $60 \%$ since 2000, respectively. The early symptoms of Malaria are very non-specific and variable (World Malaria Report, 2015). According to WHO, 
malaria is endemic in 108 countries including India. Karnataka being a vast semi-urban state with rich irrigated lands and good monsoon has promoted growth of mosquitoes and disease transmission. Malaria is very common during monsoon and post-monsoon period (Anirudh R. Acharya, 2013).

Parasite based diagnosis is increasing, most suspected cases of malaria are still not properly identified, but the treatment has to be started immediately in order to avoid complications. This has resulted in poor disease diagnosis and overuse of anti-malarial drugs (Deepika Verma et al., 2014). The commonly employed method for diagnosis of malaria involves the microscopic examination of Leishman stained blood films (Anirudh R. Acharya, 2013). An expert microscopist can detect 20-40 parasites/ $\mu$ on standard blood films. Although a thick peripheral blood smear (PBS) allows identification of the plasmodial parasite and stages, the technique is laborious, time consuming and requires a well-trained microscopist for accurate identification (Bruce-Chwatt, 1984; Pinto et al., 2001; Manjunatha et al., 2011).

Since 2010 WHO has recommended that all persons with suspected malaria in all settings should undergo malaria diagnostic testing by either microscopy or Rapid diagnostic tests (RDT) (Yasmeen Khatib et al., 2016; World Health Organization, 2011). Peripheral blood smear examination (PBS) is done routinely. However, PBS during febrile period is essential for demonstration of parasite.

In most cases, sample collection during afebrile period or at inappropriate timings results in smear negative reports. PBS has limitation like it detects only when 40-60 parasites $/ \mu \mathrm{l}$ of blood are present. Hence, antigen detection tests or others would be ideal for early diagnosis or to confirm malaria (Pinto et al., 2001).
In recent years, numerous quick and new techniques for malaria diagnosis have been developed, one such being the rapid diagnostic test. The antigen detected is histidine rich protien-2 (HRP-2) and enzymes detected are plasmodium lactate dehydrogenase (pLDH) and pan-specific aldolase. All these techniques vary in their sensitivity, specificity, positive and negative predictive values. The objective of the study was to estimate the prevalence of malaria and compare Leishman stained thick and thin PBS with that of Rapid diagnostic test in the diagnosis of malaria.

\section{Materials and Methods}

This Cross sectional study was conducted in the Department of Microbiology, VIMS Ballari during monsoon and post monsoon period during June 2016 to October 2016. The study group comprised of patients with pyrexia of Unknown origin (PUO) referred from outpatient/inpatient departments to the Microbiology laboratory for the diagnosis of malaria. The study included patients of either gender age varied between 1-80 years. And patients diagnosed with dengue fever, ricketsial fever were excluded.

\section{Sample collection}

$1 \mathrm{ml}$ venous blood collected into EDTA bulbs and subjected to antigen detection using commercially available kits (SD Bioline) and then PBS was prepared and stained using Leishman stain.

\section{Thick and thin blood smears}

Thick and thin blood smears were prepared as per the standard method. The smears were stained with Leishman's stain. Approximately 80-100 fields were examined over 8-10 minutes by an experienced microscopist. (Manjunatha et al., 2011) Negatives should not be reported until 200 oil immersion fields 
have been examined and Additional specimens should be examined at 12-hour intervals for a subsequent 36 hours.

\section{Antigen detection using}

HRP II Ag of Pf and pLDH of Pv: Commercially available antigen detection kit (SD Bioline) was used. The test was done using EDTA anticoagulated blood as per standard operating procedure (SOP) given by manufactures. Interpretation of the test result was done as below:

When only one pink- purple band appears in the control window marked as " $\mathrm{C}$ " the test Considered to be negative.

When one control band and another one bands appear only at region of "P.f" the test considered to be positive for $P$. falciparum.

When one control band and one pink purple band appear only at region "P.v" the test considered to be positive for $P$. vivax.

When one control band and another two bands appear at regions "P.f" and "P.v" the test considered to be mixed infection of P.f and P.v.

When no control band appears on device the test should be considered invalid.

\section{Statistical Analysis}

The data is expressed in descriptive statistics as frequency and percentage.

\section{Results and Discussion}

2979 samples were screened for malaria parasite by thick and thin Leishman stained peripheral blood smear method and RDT antigen detection test. The study flow chart is depicted in Figure 1. The Month wise Trend of sample outflow during Monsoon \& Post-
Monsoon Periods is shown in table 1. During monsoon period 1616 (54.24\%) samples were tested whereas in the post monsoon 1363 $(45.75 \%)$ samples were tested. The Age-wise distribution of positive cases is shown in table 2 . The age group commonly affected was 2040 years $(75 \%)$ with gender ratio of $3: 1(\mathrm{M}: \mathrm{F})$ showing male preponderances $(75 \%)$. The gender-wise distribution of positive cases is shown in Table 3. Geographic prevalence estimated Ballari distict (urban) was $0.74 \%$.

Malarial parasite was detected in $12(0.74 \%)$ cases during the monsoon period. Out of a total of 12 positive cases, while $3(25 \%)$ samples were positive by peripheral smear and 12(100) were detected positive by RDT. In the Post monsoon period none of the patients have shown positivity either by slide method or Rapid diagnostic methods.

Rapid detection and early treatment are pre requisite in the management of malaria. Peripheral blood smear method is simple, least expensive, labor intensive, time consuming and therefore delay the diagnosis. PBS smears are considered to be the 'gold standard' in diagnosis. However, the accurate species differentiation and quantification of parasitemia is possible only when performed by skilled microscopist. (Jafar Ebrahim et al., 2013) Apart from clinical and microscopic examination, many rapid diagnostic tests have been developed which are rapid, simple and easy to interpret. Most frequently, they deploy a dipstick or antigen card tests.

Prompt and accurate diagnosis of malaria is a key to effective management because it helps in early start of appropriate antimalarial drug to prevent complications In view of this, new rapid diagnostic techniques have been developed and evaluated in recent years. In the present study, the prevalence of malaria in Ballari was assessed using simple microscopic and rapid diagnostic methods. 
Fig.1 Study flow chart

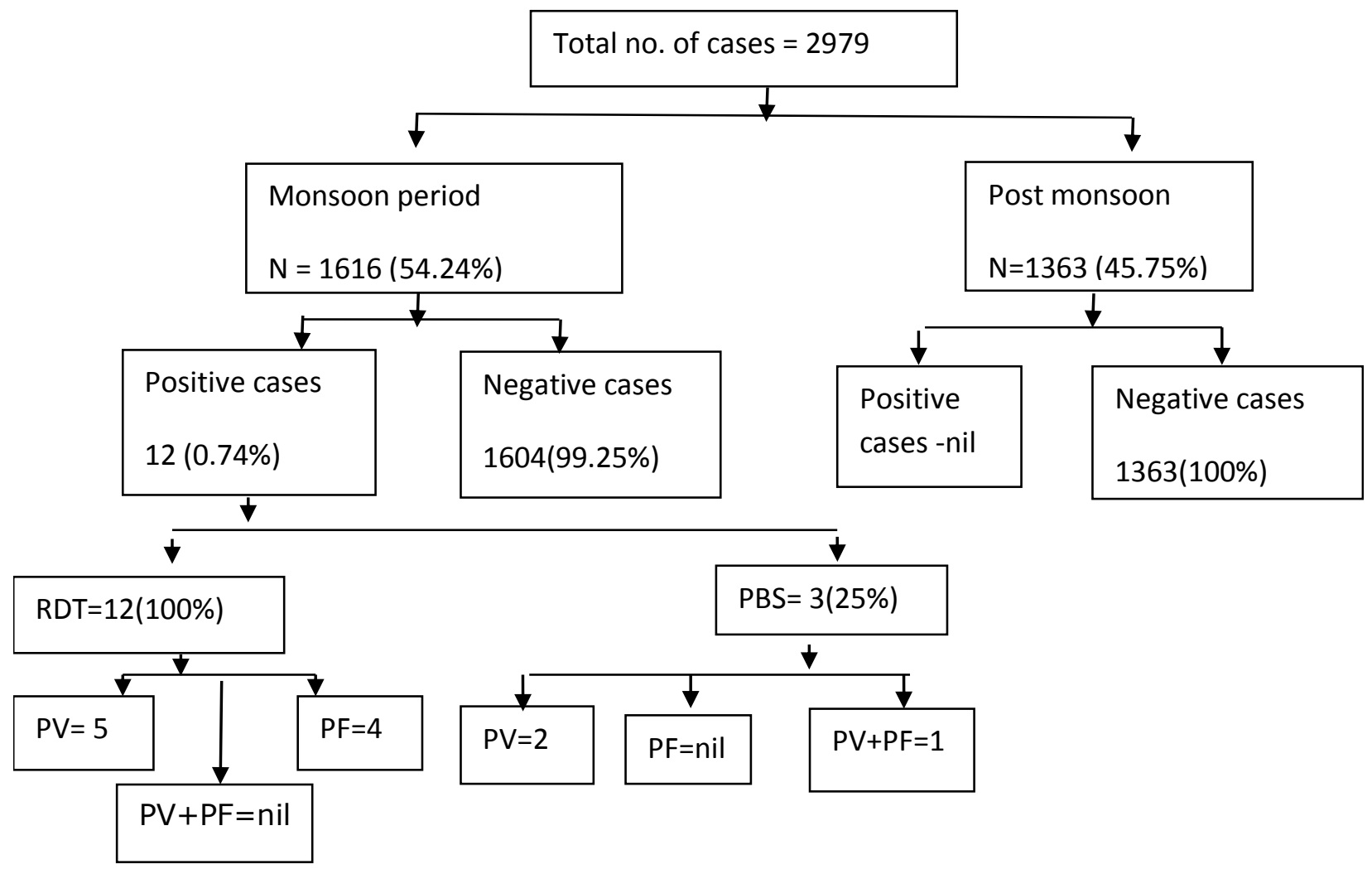

Table.1 Month wise Trend of sample outflow during monsoon and post-monsoon periods

\begin{tabular}{|c|c|c|c|c|}
\hline & Months & Total cases & Positives & Negative \\
\hline \multirow{3}{*}{$\begin{array}{l}\text { Monsoon period } \\
(n=1616)\end{array}$} & June & 370 & 04 & 366 \\
\hline & July & 566 & 05 & 561 \\
\hline & August & 680 & 03 & 677 \\
\hline \multirow{2}{*}{$\begin{array}{l}\text { Post } \quad \text { Monsoon } \\
\text { period }(n=1363)\end{array}$} & September & 662 & Nil & 662 \\
\hline & October & 701 & Nil & 701 \\
\hline Total & & 2979 & 12 & 2967 \\
\hline
\end{tabular}

Table.2 Age-wise distribution of positive cases

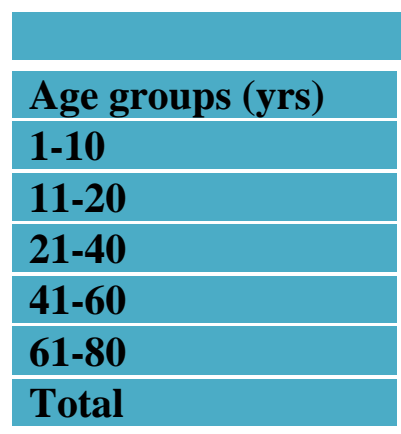

\begin{tabular}{|l|l|l|l|}
\hline \multicolumn{1}{|c|}{ RDT } & \multicolumn{2}{c|}{ PBS } \\
\hline P. vivax & P. falciparum & P. vivax & P. falciparum \\
\hline-- & -- & -- & -- \\
\hline 02 & 01 & -- & -- \\
\hline 03 & 03 & 02 & 01 \\
\hline-- & -- & -- & -- \\
\hline-- & -- & -- & -- \\
\hline $\mathbf{0 5}$ & $\mathbf{0 4}$ & $\mathbf{0 2}$ & $\mathbf{0 1}$ \\
\hline
\end{tabular}


Table.3 Gender-wise distribution of Positive cases

\begin{tabular}{|l|l|l|l|l|l|}
\hline & \multicolumn{2}{|c|}{ RDT } & \multicolumn{2}{|l|}{ PBS } & Total \\
\hline Gender & P. vivax & P. falciparum & P. vivax & P. falciparum & \\
\hline Male & 04 & 03 & 02 & -- & $09(75 \%)$ \\
\hline Female & 01 & 01 & & 01 & $03(25 \%)$ \\
\hline Total & $\mathbf{0 5}$ & $\mathbf{0 4}$ & $\mathbf{0 2}$ & $\mathbf{0 1}$ & $\mathbf{1 2}$ \\
\hline
\end{tabular}

The Rapid Antigen Detection Assay results indicated a prevalence of $12(100 \%)$ while microscopy indicated the total prevalence of 3 $(25 \%)$ out of the 100 persons that were examined (Moody, 2002; Singh et al., 2013). The sensitivity of thin smear found to be lowest $(25 \%)$ which may be because of the undefined ring stage of the parasite. While (Parija and Praharaj, 2011) reported a very low sensitivity of $(54.8 \%)$ leishman stained thin smear. Leishman stained thin blood smear detects malarial parasite only when there are 50 parasite/ $\mathrm{ml}$ of blood. Even though this method can be used for species identification of malarial parasite, Still the limitation of rapid diagnostic methods like failure of the parasites to express the antigen due to deletion of the gene pfhrp2 (Koita et al., 2012), anti Pf-HRP2 antibodies in humans or storage conditions remained (Koita et al., 2012)

There was an increase in trend of fever of unknown origin during monsoon and post monsoon period. However, the results of positive cases are lower during this monsoon and post monsoon seasons. The geographic prevalence was nil during the post monsoon period. This may be attributed to the fact that the present study was carried out during dry season when stagnant water bodies have been dried up and the breeding sites of mosquitoes interrupted or progress in the control of malaria through the use of insecticide by Municipal Corporation. The result clearly indicated that Rapid antigen detection test had higher sensitivity and specificity compared to PBS. Our study results contradict with study by (Moody, 2002; Singh et al., 2013). The prevalence of malaria parasite by both methods showed that, age group 21-40 years had the greatest risk of infection followed by 11-20 years (Table 2). The prevalence of malaria parasite by gender as shown in both methods was higher in males than in females (See Table 3). This sex distribution showed that males are more prone to the infection which is in accordance with the Nwaorgu and Orajiaka (2011) and Okafor and Oko-Ose, (2012) who reported higher prevalence in males in different parts of the country.

This study revealed declining trend of malaria in 2016. Rapid antigen detection kits are more specific and sensitive in detection of the malarial parasites especially in endemic areas even with low parasitaemia. Thus RDT can be a promising alternative to microscopy. But still microscopy remains the gold standard method for malaria diagnosis considering the cost and availability.

\section{Acknowledgements}

All the staff members of Microbiology Department, VIMS.

\section{References}

Anirudh R Acharya, Jhansi Lakshmi Magisetty, Adarsha Chandra VR. Chaithra BS, Taiyaba Khanum and Vijayan VA. Trend of malaria incidence in the state of Karnataka, India for 2001 to 2011. Archives of Applied Science Research 2013; 5(3):104-111. 
Bruce-Chwatt LJ. DNA probes for malaria diagnosis. Lancet 1984; 1:795.

Deepika Verma, Sachin Kishore, Siddiue ME. Comparative evaluation of various tests for diagnosis of concurrent malaria and typhoid fever in a tertiary care hospital of northern India. Journal of Clinical and Diagnostic Research. 2014; 8(5):41-44.

Jafar Ebrahim, J., I. Al-Ghonaim Mohammed, Maghram Al-Amri, H. Abdel-Ghaffar Mamdouh1, N. Ramprasad, R. Farhan Khan. Comparative Study of Thick Smear, Thin Smear, QBC and Antigen Card Test in Diagnosis of Malaria. Int. J. Pure Appl. Sci. Technol 2013; 17(1): 54-59.

Koita OA, Doumbo OK, Ouattara A, Tall LK, Konaré A. False-negative rapid diagnostic tests for malaria and deletion of the histidine-rich repeat region of the hrp2 gene. Am J Trop Med Hyg 2012; 86: 194-198.

Manjunatha S, Preeti BM, Basavaraj VP. Comparative Study of Peripheral Blood Smear, QBC and Antigen Detection in Malaria Diagnosis. Journal of Clinical and Diagnostic Research 2011; 5(5):967-969.

Moody, A. Rapid Diagnostic Tests for Malaria Parasites. Clinical Microbiology Reviews 2002; 15(1): 66 $-78$.

Nwaorgu, O.C. and Orajaka, B. N. Prevalence of malaria among Children 1-10 years old in Communities in Awka North Local Government Area, Anambra State, South East Nigeria. International Multidisciplinary Journal Ethiopia
2011. 5(5): $264-281$.

Okafor, F. U. and Oko-Ose, J. N. Prevalence of malaria infections among children aged six months to eleven years (6 months-11 years) in a tertiary institution in Benin City, Nigeria. Global Advanced Journal and Medical Sciences 2012; 1(10): 273 - 279.

Parija SC, Praharaj I. Drug resistance in malaria. Indian J Med Microbiol 2011; 29: 243-8.

Pinto MJW, Rodrigues SR, Desouza R, Verenkar MP. Usefulness of quantitative buffy coat blood parasite detection system in diagnosis of malaria. Indian J Med Microbiol 2001; 19:219-21.

Singh, R., Abdullahi, K., Mohammad, D. A., Bunza, M. D. A., Njoku, C. N., Singh, S., Usman, N. S., and Kaitholia, K. Comparative Diagnosis of Falciparum Malaria Infections by Microscopy, Two RDTs and Nested PCR in the Three States of North- western Nigeria. Journal Biology Agriculture and Healthcare 2013; 3(5): 31- 38.

World Health Organization, Universal Access to Malaria Diagnostic Testing: an Operational Manual, WHO, Geneva Switzerland, 2011.

World Malaria Report, Geneva Switzerland: WHO Press; 2015

Yasmeen Khatib, Sanjay Gulhane, Karen Sequeira. Correlation and comparative evaluation of Thick smear, thin smear and Antigen detection test in the diagnosis of Malaria. Sch. J. App. Med. Sci 2016; 4(5):1770-1773.

\section{How to cite this article:}

Vishalakshi, B., J. Mariraj, S. Liba and Krishna, S. 2018. Cross Sectional Study Comparing Peripheral Blood Smear and Rapid Antigen Detection Test in Diagnosis of Malaria Conducted During Monsoon and Post Monsoon Period at Ballari, India. Int.J.Curr.Microbiol.App.Sci. 7(02): 255-260. doi: https://doi.org/10.20546/ijcmas.2018.702.033 